\title{
Failure Investigation of WB-57 Aircraft Engine Cowling
}

\author{
J. Martinez ${ }^{1}$, T. Gafka ${ }^{2}$, J. Figert ${ }^{1}$ \\ ${ }^{1}$ Structural Engineering Division, NASA Lyndon B. Johnson Space Center, Houston, TX 77058 \\ ${ }^{2}$ Aircraft Operations Division, NASA Lyndon B. Johnson Space Center, Houston, TX 77058
}

The NASA Johnson Space Center (JSC) in Houston, Texas is the home of the NASA WB-57 High Altitude Research Program. Three fully operational WB-57 aircraft are based near JSC at Ellington Field. The aircraft have been flying research missions since the early 1960's, and continue to be an asset to the scientific community with professional, reliable, customer-oriented service designed to meet all scientific objectives.

The NASA WB-57 Program provides unique, high-altitude airborne platforms to US Government agencies, academic institutions, and commercial customers in order to support scientific research and advanced technology development and testing at locations around the world. Mission examples include atmospheric and earth science, ground mapping, cosmic dust collection, rocket launch support, and test bed operations for future airborne or spaceborne systems ${ }^{[1]}$.

During the return from a 6 hour flight, at 30,000 feet, in the clean configuration, traveling at 175 knots indicated airspeed, in un-accelerated flight with the auto pilot engaged, in calm air, the 2-man crew heard a mechanical bang and felt a slight shudder followed by a few seconds of high frequency vibration. The crew did not notice any other abnormalities leading up to, or for the remaining 1 hour of flight and made an uneventful landing. Upon taxi into the chocks, the recovery ground crew noticed the high frequency long wire antenna had become disconnected from the vertical stabilizer and was trailing over the left inboard wing, and that the left engine upper center removable cowling panel was missing, with noticeable damage to the left engine inboard cowling fixed structure. The missing cowling panel was never recovered.

Each engine cowling panel is attached to the engine nacelle using six bushings made of 17-4 PH steel. The cylinder portions of four of the six bushings were found still attached to the aircraft (Fig 1). The other two bushings were lost with the panel. The other four bushings exhibited ratchet marks (multiple fatigue origins) which initiated in the sharp radius of the flange/cylinder fillet and were observed 300 degrees around the flange perimeter (Fig 2-3). Low stress, high cycle fatigue (HCF) was observed on the fracture surfaces of all four bushings $^{[2]}$ (Fig 4).

To improve the cowling panel joint design and enable return to flight, new cowling bushings with thicker flanges and a larger machined flange/cylinder fillet radius were installed on all cowling panels. In addition, a spacer was added to the joint to achieve the proper stack tolerance. Finally, a time change requirement for all cowling bushings was instituted.

\section{References:}

[1] http://jsc-aircraft-ops.jsc.nasa.gov/wb57/

[2] ASM Handbook, Vol 11, pp 102-135 


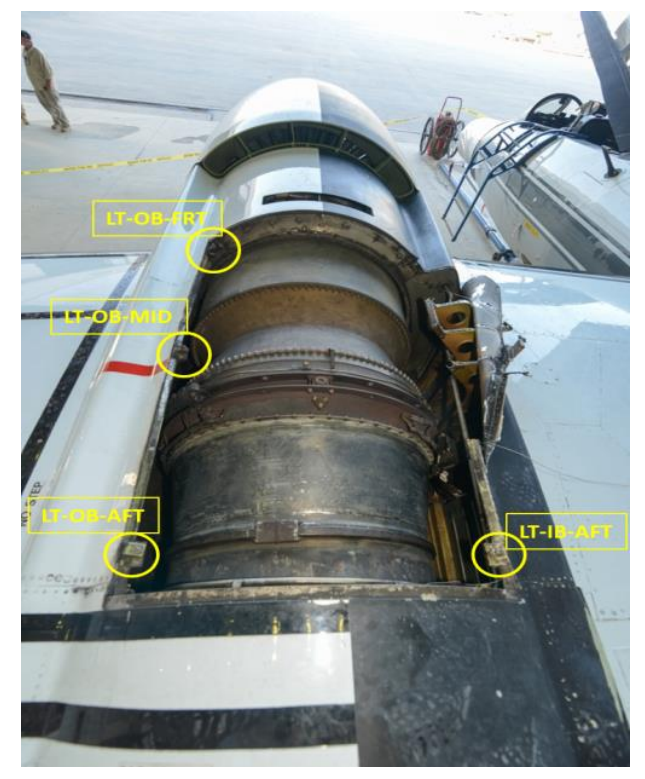

Fig 1. WB-57 Aircraft Left Engine w/Missing Cowling. Location of four remaining bushings circled in yellow.

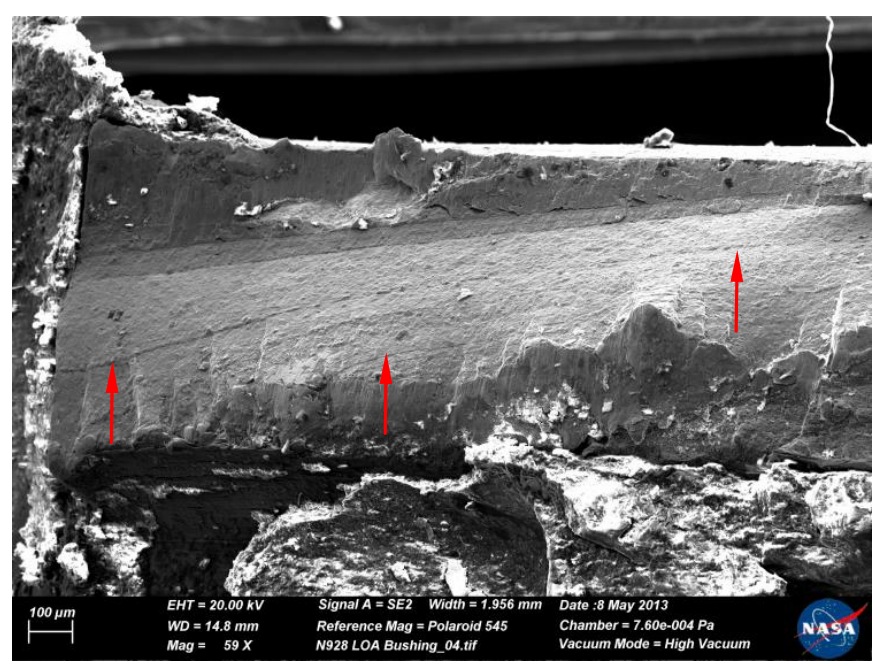

Fig 3. SEM of Failed Engine Cowling Bushing (ratchet marks at red arrows)

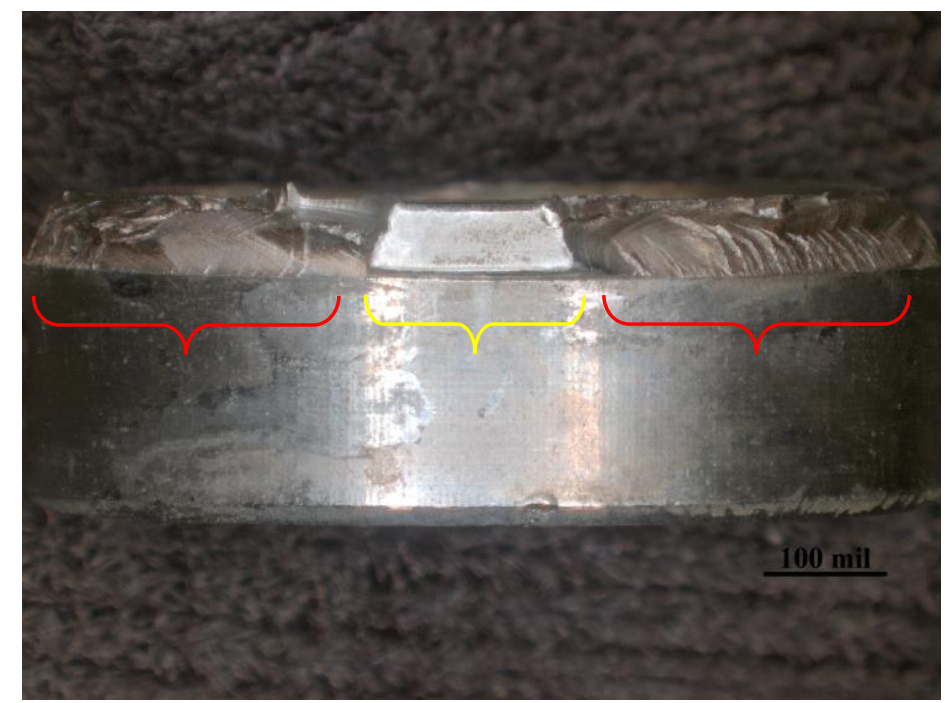

Fig 2. Photo of Failed Engine Cowling Bushing. Fatigue fracture zone in red, unfractured zone in yellow.

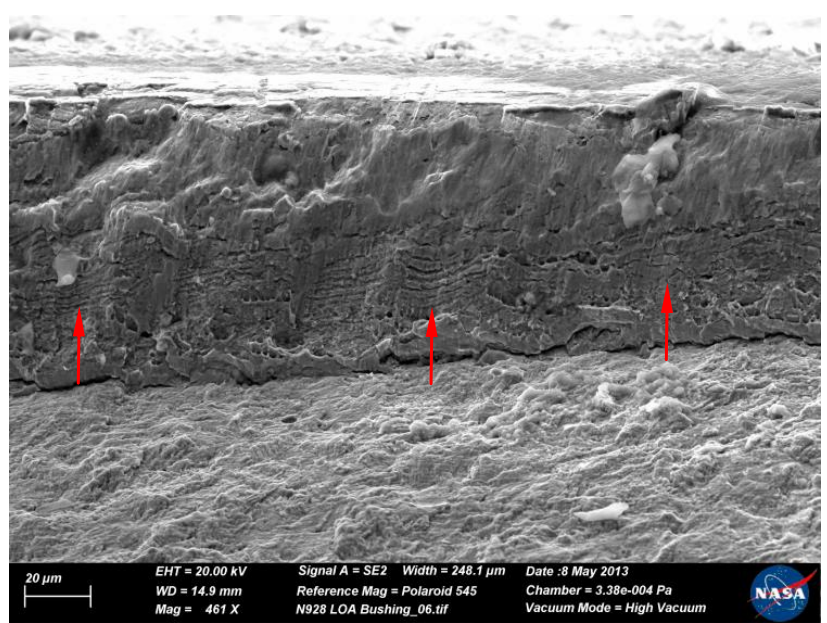

Fig 4. SEM Image of HCF Striations (red arrows) 